\title{
FALTA DE SENTIDO DE VIDA Y SUICIDIO: ¿EN QUÉ CIRCUNSTANCIAS EL PATERNALISMO MÉDICO PUEDE ESTAR JUSTIFICADO?
}

\begin{abstract}
Mariana Espínola-Nadurille, José Antonio Sánchez-Barroso², Ricardo Páez-Moreno³, María Alejandra Sánchez-Guzmán ${ }^{4}$, Jesús Ramírez-Bermúdez ${ }^{1}$

Resumen: Objetivos. Descripción de un caso clínico abordado a través de un enfoque bioético. Materiales y métodos. Por medio de la deliberación moral, se aclararon los hechos relevantes y se identificaron los problemas morales; se seleccionó el problema ético central y se plantearon cursos de acción. Resultados y conclusiones. En este caso clínico la solución más prudente incluyó el paternalismo justificado. La práctica de la deliberación moral es indispensable, porque las decisiones frecuentemente rebasan la técnica médica y exigen la ponderación de valores morales.
\end{abstract}

Palabras clave: deliberación moral, suicidio lúcido, paternalismo justificado, violencia y bioética

\section{Lack of sense of life and suicide: In which circumstances medical paternalism may be justified?}

Abstract: Aims: Description of a clinical case addressed by using a bioethical focus. Materials and methods: Relevant facts were clarified and moral problems were identified by moral deliberation; main ethical problem was selected and courses of action were suggested. Results and conclusions: In this clinical case the most prudent solution was justified paternalism. The practice of moral deliberation is essential since decisions frequently surpass medical techniques and demand the deliberation of moral values.

Key words: moral deliberation, lucid suicide, justified paternalism, violence and bioethics

\section{Falta de sentido de vida e suicídio: em que circunstâncias o paternalismo médico pode estar justificado?}

Resumo: Objetivos. Descrição de um caso clínico abordado através de um enfoque bioético. Materiais e métodos. Por meio da deliberação moral foram esclarecidos os fatos relevantes e se identificaram os problemas morais; se selecionou o problema ético central e se apresentou cursos de ação. Resultados e conclusōes. Neste caso clínico a solução mais prudente incluiu o paternalismo justificado. A prática da deliberação moral é indispensável porque as decisões frequentemente ultrapassam a técnica médica e exigem a ponderaçáo de valores morais.

Palavras-chave: deliberação moral, suicídio lúcido, paternalismo justificado, violência e bioética

\footnotetext{
${ }^{1}$ Unidad de Neuropsiquiatría, Laboratorio de Salud Pública y Epidemiología, Instituto Nacional de Neurología y Neurocirugía "MVS", Facultad de Medicina, Universidad Nacional Autónoma de México, México

${ }^{2}$ Facultad de Derecho, Universidad Nacional Autónoma de México, México Correspondencia: jasanchez@up.edu.mx

${ }^{3}$ Programa de Maestría y Doctorado en Bioética, Universidad Nacional Autónoma de México, México

${ }^{4}$ Laboratorio de Violencia y Género, Instituto Nacional de Neurología y Neurocirugía “MVS”, México
} 


\section{Introducción}

En las últimas décadas, la práctica de la medicina ha sido objeto de múltiples transformaciones. La supremacía de la vida se ha cuestionado ante la realización de otros valores igualmente importantes, tales como la libertad y la justicia. La relación clínica, que antes se regía por el paternalismo clásico, ahora es una relación más participativa y propositiva. Hoy el paciente es considerado autónomo y capaz de tomar decisiones en todo aquello que afecte su proyecto personal de vida. De manera semejante, a partir de la modernidad se define el papel del Estado en la determinación de lo justo, que en bioética sería la atención sanitaria que, en justicia, le compete otorgar a sus ciudadanos para evitar problemas de abandono.

El objetivo es analizar, desde la bioética deliberativa, el caso de una paciente registrado en la Unidad de Neuropsiquiatría del Instituto Nacional de Neurología y Neurocirugía "MVS", Facultad de Medicina, Universidad Nacional Autónoma de México, en el que se presentan problemas éticos, clínicos y sociales. Es necesario un trabajo interdisciplinario para que cada una de las áreas del conocimiento que aquí confluyen (bioética, derecho, filosofía, ciencias sociales, psicología y medicina) aporten soluciones que verdaderamente sean factibles e integrales. Por la naturaleza de la práctica médica, los casos clínicos frecuentemente presentan dilemas éticos; sin embargo, se suelen abordar sin sustento teórico metodológico que guíe la deliberación. Presentamos de manera detallada el uso del método de la deliberación moral para construir recomendaciones prudentes.

La pregunta central es en qué circunstancias el paternalismo médico puede estar justificado, aun cuando se actúa en contra de la voluntad del paciente y de su familia, ante una situación clínica de incertidumbre y un contexto social de abandono.

\section{Material y métodos}

Se realizaron entrevistas en profundidad con la paciente, las que fueron transcritas y analizadas con etnograph, para rescatar los elementos más destacados en el método de deliberación moral.
El método para analizar el caso es el deliberativo propuesto por Diego Gracia, que tiene como propósito considerar, en un primer momento, los deberes morales aplicables al caso concreto (momento deontológico) y, en un segundo, las circunstancias particulares y consecuencias previsibles (momento teleológico); es decir, establecer racionalmente un vínculo entre valores y hechos, con el fin de ofrecer soluciones moralmente buenas y prudentes $y$, además, fácticamente aplicables (1).

\section{Presentación del caso}

Mujer (“Ángeles") de 34 años, de nivel socioeconómico bajo, solo con habilidades de lectoescritura, que ingresa al área de hospitalización psiquiátrica por un intento suicida en los días previos. Es traída por su madre y su padrastro. Su padre biológico se dedica al campo y su madre al hogar. Ellos se separaron cuando Ángeles tenía 2 años.

En su infancia Ángeles vivió en provincia bajo el cuidado de un tío, quien abusó física y verbalmente de ella. Después de algunos años fue llevada a vivir a la ciudad de México con su madre y su padrastro, quien es 20 ańos mayor que su madre.

El padrastro comenzó a abusar sexualmente de Ángeles cuando esta tenía 12 años. La madre, al enterarse, se vuelve hostil hacia ella.

A los 13 años, Ángeles se embaraza, como consecuencia de los repetidos abusos sexuales. Al término del embarazo presenta eclampsia y, desde entonces, padece epilepsia. Por decisión de la madre y el padrastro, el hijo es dado en adopción al nacer, sin consultar a Ángeles.

A su llegada al hospital, Ángeles manifiesta: "durante toda mi vida he tenido ganas de matarme; sin embargo, hasta ahora me atreví, siempre me he sentido una mujer asquerosa”.

Durante el tiempo que Ángeles ha vivido con su padrastro ha sufrido abuso sexual. Ahora él tiene 76 años y disfunción eréctil; no obstante, aún mantiene diferentes formas de contacto sexual con ella. De acuerdo con el testimonio de Ángeles, actualmente además la agrede verbalmente: "estás fea, antes eras bonita", "no te das cuenta, 
no has hecho nada de tu vida, no tienes nada". Su madre nunca ha intervenido para evitar el maltrato sexual o psicológico.

Ocho días previos a su internamiento presenta intento suicida ingiriendo ocho tabletas de clonazepam. Al preguntarle la causa, responde que su vida no tiene sentido: "no sé ni para qué estoy aquí, perdí a mi hijo, no he hecho nada de mi vida, no tengo por qué estar viva”.

En los primeros días de internamiento solicita su alta voluntaria "para matarse", de acuerdo con sus propias palabras. La madre y el padrastro también la solicitan. El médico tratante considera que existe un problema bioético por la salud mental de la paciente, la violencia y las condiciones socioculturales en las que vive. El caso se presenta en la comisión asistencial de ética del Instituto para determinar si se justifica un internamiento involuntario, con el fin de proteger la vida de la paciente, así como los posibles cursos de acción posteriores al internamiento. La mayoría de los miembros de la comisión consideran que la paciente goza de autonomía, aunque tres de ellos tienen duda razonable.

El comité concluye que un consentimiento informado acerca de su hospitalización no sería válido, ya que la voluntariedad de Ángeles está mermada por la coacción y la manipulación por parte de sus padres (por ejemplo, el padrastro la amenaza con encerrarla de manera permanente si no la obedece) y, además, por los miedos e inseguridades ante su propia situación (por ejemplo, manifiesta: "quedarme sola es mi miedo más grande"). En consecuencia, se decide un internamiento involuntario y se solicita al Centro de Atención a Victimas de Violencia (CAVI) del DF apoyo para el manejo integral del caso: abordaje psicológico y alojamiento en algún albergue para mujeres víctimas de violencia. Cuando se le informa a la paciente acerca de la posibilidad de ofrecerle alojamiento en un alberge, ella se muestra entusiasta y manifiesta: "si pudiera comenzar de nuevo, a lo mejor se me quitarían las ganas de morir”.

En la evolución intrahospitalaria se corrobora que la paciente no se encuentra en un cuadro depresivo, pues desde el segundo día de internamiento no se observaron los síntomas que integran ese cuadro.

Aun cuando se prohíben visitas y llamadas de familiares, en el periodo en que se consigue un albergue, la paciente realiza llamadas por cobrar a casa. Al no ver evolución de su caso, insiste en que quiere irse con su familia, reitera que su miedo más grande es quedarse sola: "ahora me están tratando bien, seguro sí me quieren". Sin embargo, en un par de ocasiones más vuelve a verbalizar la posibilidad de suicidio al egreso "por falta de sentido de vida", de acuerdo con sus palabras.

El CAVI informa que la aceptación de alojamiento en algún albergue se dificulta, ya que ante la presentación de este tipo de casos, (los albergues) manifiestan sentirse "rebasados" por la enfermedad neurológica y el gesto suicida previo, aun cuando se les informa que la enfermedad epiléptica se encuentra bajo control con medicamentos y que la posibilidad de un nuevo intento suicida se considera baja, de encontrarse la paciente en un contexto social protegido.

Ángeles solicitó en repetidas ocasiones su alta voluntaria y esta no fue otorgada. Se intentó la persuasión, informándole los beneficios del internamiento. Su respuesta era ambivalente: por días estaba de acuerdo y en otros momentos reiteraba su petición de alta. Después de 30 días de hospitalización, es egresada directamente a un albergue para mujeres en situación de violencia.

\section{Hechos relevantes e identificación de proble- mas morales}

1. Infancia en un seno familiar disfuncional.

2. Víctima de violencia física, psicológica y sexual desde su infancia hasta la fecha.

3. Por las repetidas violaciones, queda embarazada a los 13 años.

4. Se dio en "adopción" a su hijo sin su consentimiento.

5. Manifiesta expresamente que tiene deseos de matarse.

6. Tiene sentimientos encontrados con sus familiares.

7. Hay un intento de suicidio.

8. Existe duda sobre su autonomía. 
9. Ella y sus familiares han pedido el alta voluntaria.

10. La paciente no tiene otros sentidos, caminos o escenarios de vida.

11. Se evidencia un contexto de pobreza económica, educativa y moral. La paciente no cuenta con otra red social, a excepción del agresor y la madre, quien ha permitido el abuso a Ángeles y probablemente a ella misma.

12. El caso se inserta en un contexto sociocultural tolerante a la violencia doméstica y de género.

\section{Elección del problema}

Este caso, como prácticamente todos los que son objeto de análisis bioético, presenta una pluralidad de problemas; es decir, gravitan alrededor varios problemas morales o de otro tipo (sociales, económicos, jurídicos, institucionales, etc.), que pueden ser objeto de estudio. Sin embargo, la deliberación moral exige que se elija uno de ellos (el principal o el que escoja la persona que presenta el caso), de modo de hacer más explícito el conflicto de valores y más fácil la evidencia de los cursos óptimos de acción.

Consideramos que el Problema Ético Central (PEC) del caso que ahora nos ocupa es: el centro hospitalario, ¿puede mantener internada a la paciente, aun en contra de su voluntad y la de sus familiares, por el temor fundado a un suicidio?

En relación con este, si no existe depresión intercurrente:

1. ¿Qué acciones puede y debe realizar la institución hospitalaria para disminuir el riesgo suicida?

2. La paciente, ¿ ¿tiene realmente competencia?

3. Si la paciente no es competente, ¿pueden los familiares decidir por ella?

4. ¿Qué papel le corresponde al INNN y cuál al deber de asistencia social del Estado?

Antes de identificar los valores en conflicto, es necesario esclarecer cuatro temas en los que se enfocará propiamente la deliberación: diferencia entre competencia y capacidad; relativo al paternalismo médico; relativo al suicidio, y justicia y deber del Estado de atender situaciones de exclusión.
En cuanto al primero, Beauchamp y Childress, en "Principles of biomedical ethics" (2), establecen una serie de condiciones para que la acción proveniente de una persona sea catalogada como autónoma: i) que se realice intencionalmente, ii) que se realice con entendimiento, y iii) que se realice sin influencias de control que determinen la acción (coacción externa —en caso de presión por otras personas a realizar un acto- - y coacción interna -miedo intenso a las consecuencias de ejercer una acción-, es decir, que exista voluntariedad. De estas tres, la primera no admite graduaciones, es decir, la intención para realizar un acto se tiene o no se tiene, no hay intención a medias. Solo las dos últimas pueden tener grados, ya que la persona puede tener mayor o menor grado de entendimiento o conocimiento respecto de una circunstancia específica. De igual modo, la libertad puede estar más o menos determinada por factores externos, de forma que no es requisito sine qua non un grado absoluto de entendimiento y libertad, aunque constituye el ideal.

Ser respetado como autónomo supone reconocer el derecho de la persona a realizar sus elecciones con criterio propio, sobre la base de valores y creencias personales; por ende, no supone compartir una fundamentación metafísica de la autonomía, sino simplemente reconocer y aceptar un derecho de todo individuo a actuar bajo determinadas condiciones y circunstancias.

El principio de autonomía implica no solo la obligación de no intervenir en los asuntos de otras personas, sino también la de asegurar las condiciones para que su elección sea independiente, mitigando los miedos y toda circunstancia que pueda dificultarla o impedirla. Por ello, este principio puede formularse tanto positiva como negativamente(3).

La forma en que se han hecho efectivos el respeto y la promoción del principio de autonomía es en el "consentimiento informado"(4). Por otro lado, la capacidad no puede ni debe confundirse con la competencia. La capacidad es propiamente un concepto legal. La competencia, en cambio, un concepto directamente relacionado con el principio de autonomía en la relación clínica. Una persona en un mismo momento puede tener 
capacidad para realizar determinadas acciones autónomas, pero no la suficiente competencia para decidir. La competencia no es constante, sino específica para cada decisión; además, no solo puede cambiar en el tiempo (estado mental, medicación, cansancio), sino también verse influida por el estado afectivo (sensación de frustración o depresión).

La máxima de la competencia en relación con el principio de autonomía es: "a medida que aumenta el riesgo de la decisión, el nivel de competencia exigible es más elevado", es decir, "a mayor riesgo, criterios más estrictos". Esto se manifiesta en una escala móvil de competencia(5). Cuando una persona no se considera suficientemente competente para decidir se busca la sustitución de toma de decisiones. El fin de buscar otra instancia de decisión y proceder con la intervención terapéutica es "restaurar" la autonomía del paciente, para que posteriormente sea él el agente que tome las decisiones subsecuentes. Ante la necesidad de toma de decisiones por sustitución, se elige a los familiares cercanos o "proxies", considerándose que ellos son los que conocen mejor a la persona y pueden tomar la decisión: i) de acuerdo con los deseos del paciente, si los pudo expresar antes de encontrarse en la situación de incompetencia; ii) de acuerdo con los valores de la persona, y iii) de acuerdo con sus mejores intereses(6).

Respecto del paternalismo médico, Manuel Atienza afirma que existen dos elementos esenciales que deben concurrir en una acción o conducta para que pueda ser calificada como paternalista: i) el fin de obtener un bien para una persona o grupo de personas, y ii) no contar con la aceptación de la persona o personas involucradas, es decir, de los presuntos beneficiados por la realización de la conducta. Agrega que esta acción paternalista podrá ser éticamente justificada si: a) está realmente encaminada hacia la consecución de un bien objetivo de una persona o de una colectividad, b) los individuos o la colectividad a quien se aplica o destina la medida no pueden prestar su consentimiento por poseer algún tipo de incapacidad básica o incompetencia, y c) se puede presumir racionalmente que estos prestarían su consentimiento si no estuvieran en la situación de incapacidad o incompetencia indicada $y$, por tanto, conocieran cuál es realmente su bien(7).
La medida paternalista solo estará justificada si resulta ser un medio técnicamente adecuado para la consecución del bien perseguido y siempre que no suponga la destrucción de otros bienes de igual o similar valor.

Durkheim dice que el suicidio es todo caso de muerte, que resulte directa o indirectamente de un acto por comisión u omisión, realizado por la víctima misma, a sabiendas de que su acto provocará este resultado(8). Cohen considera que "en todas las definiciones de suicidio existe el supuesto común del reflejo del acto suicida, por el cual quién muere y quién mata es la misma persona" (9).

El suicidio se asocia frecuentemente a trastornos como esquizofrenia, enfermedad bipolar y depresión. El 70\% de las personas que cometen suicidio son víctimas de una enfermedad mental. En muchos casos de depresión severa, la autonomía puede considerarse disminuida como consecuencia del proceso mórbido. Como ejemplo se encuentra el fenómeno de "constricción", caracterizado por la rigidez de pensamiento, concreción, lo que se conoce como "visión en túnel" y estrechamiento del panorama, etc. De tal forma que una solución posible (por ejemplo, el suicidio) se convierte en una solución única. Así, si se alivia el estado depresivo, se modificarían los procesos cognitivos estrechos y alterados.

El suicidio, o intento suicida, suele llamarse "patológico" cuando existe una enfermedad física o mental intercurrente. Pero en algunos casos, cuando no está presente una enfermedad, nos interrogamos si el suicido sería válido, porque surge de la autonomía de la persona, de su libertad de decidir acerca de su propio proyecto de vida, esto si puede existir una capacidad racional en la cual la persona decide terminar con su vida, y no solo decide su muerte, sino que la considera necesaria. Estos actos se conocen como "suicidio lúcido" (10). En estos casos, la pregunta es hasta qué grado se debe de tratar de evitar el suicidio.

La filosofía política en la modernidad ha caracterizado al Estado como el agente primario de justicia. A él corresponde regular la vida cívica a través de la creación de instituciones para la protección 
de los ciudadanos contra aquellos factores que atentan contra la dignidad humana. Le corresponde también ser garante de derechos básicos que permiten que una sociedad subsista y conviva de manera pacífica, por ejemplo, en el derecho a la atención sanitaria, entre otros. Cuando un Estado no ejerce correctamente su función, por estar sumamente debilitado, los factores estructurales de deterioro del bienestar van carcomiendo a la sociedad, tales como la pobreza, la falta de instituciones de asistencia pública, entre otros(11).

La noción de "capacidades", de Amartya Sen, es útil para entender los poderes del Estado y otras agencias y agentes. Las capacidades constituyen la libertad sustantiva para conseguir distintas combinaciones de funciones, la libertad para conseguir distintos estilos de vida, esto es, para poder decidir. Lo valorable son los diversos funcionamientos, es decir, las diversas cosas importantes que una persona consigue hacer o ser en su vida real. Cuando no se ofrecen las condiciones básicas para tener capacidades, no puede hablarse de libertad(12).

Cuando el Estado no es competente para proteger a sus ciudadanos y cubrir sus derechos básicos, otras instituciones deben cubrir estas necesidades básicas, entre ellas, los hospitales públicos, cuya función primordial no es ejercer la justicia, pero pueden suplir déficits sociales a través de su labor de beneficencia.

En relación con la institución médica y el suicidio, habría que considerar no solo el principio de beneficencia, sino también el principio de justicia, conformado también por acciones beneficentes. Desde el punto de vista liberal, la justicia implica respetar la autonomía de los otros, el deber de no interferir con las decisiones del otro. Sin embargo, se corre el riesgo de justificar un individualismo larvado que termina por abandonar al otro a su suerte. Según Daniel Callahan, una buena sociedad (y sus instituciones) debe reconocer las obligaciones de ayudar a otros; sus miembros deben tener virtudes tales como compasión, cuidado, amor y solidaridad(13).

Desde una ética que, además de la autonomía, considere justo velar por el bienestar del otro, cabe preguntarse: ¿qué lleva a alguien intentar a quitarse la vida? ¿No hay responsabilidad social y familiar detrás de la petición de suicidio? ¿Qué hacer para evitar este mal? El modelo sociogénico (más allá de la psicopatología individual) considera al suicidio propiciado por causas sociales. Se mira al suicidio como una estrategia de respuesta pero no en un nivel individual, sino como conformidad ante las estructuras sociales y reacción ante los roles que una sociedad crea(14).

El tema de la violencia doméstica y de género está entrelazado con las condiciones socioculturales que avalan el maltrato como una forma de disciplinamiento, expresión amorosa o estructura familiar. No es menor para la discusión comprender este contexto, pues es probable que la reacción ambivalente de Ángeles ante la violencia esté enmarcada y determinada por esta construcción social. Las víctimas de violencia deben enfrentar su sentido común, es decir, deben reelaborar la concepción de normalidad que tienen ante el maltrato.

\section{Resultados: identificación de los valores en conflicto y cursos extremos de acción}

Una vez que se ha establecido el PEC, así como los cuatro problemas que gravitan en torno a él, corresponde identificar los valores en conflicto, para posteriormente establecer los cursos extremos de acción y los cursos óptimos.

De acuerdo con el PEC, los valores en conflicto son la vida y la libertad. Por tanto, un curso extremo de acción que gestione o realice solamente el valor "vida", sacrificando por completo la libertad, sería que Ángeles se mantenga internada en el centro hospitalario indefinidamente, aun en contra de su voluntad y la de sus familiares, hasta que verdaderamente cambie de opinión en relación con su intención de suicidarse. El otro curso extremo de acción, que gestione o realice por completo el valor "libertad" a costa de la vida, sería atender solícitamente el alta voluntaria de Ángeles sin dar seguimiento a su caso.

Por otra parte, también merece atención la beneficencia —que corresponde al INNN- y la justicia — que debe ejercer el Estado-, por su mutua dependencia para que exista una vida dig- 
na en sociedad. En este segundo caso, los cursos extremos de acción consistirían, por una parte, en confiar en la justicia (bienestar social) que debería ejercer el Estado con Ángeles, accediendo a su petición de alta y desentendiéndose el INNN de su futuro. El extremo contrario sería que el INNN sea el único agente que se encargue de Ángeles de manera indefinida y aislada.

Como es evidente, ninguno de los cursos extremos de acción resulta moralmente aceptable, ni resuelve integralmente el caso que nos ocupa. Por ello, es necesario encontrar o construir los cursos intermedios de acción (CIA) que gestionen ambos valores, con el propósito de ofrecer una solución racionalmente prudente y moralmente buena.

\section{Búsqueda de los cursos intermedios (CI)}

CI1: Considerar no competente a Ángeles por limitación en su voluntariedad, en la cual existe coacción interna ("mi miedo más grande es quedarme sola") y coacción externa (manipulación por parte de los padres); y por limitación en su autonomía, ya que no es consistente en la decisión de aceptación o rechazo del internamiento (algunos días lo acepta y en otros pide el alta voluntaria). En este caso, buscar otras instancias para la toma de decisión.

CI2: No considerar a los padres de Ángeles como personas adecuadas para la toma de decisión, por ser los causantes de la violencia y porque se asume que no tomarán la decisión de acuerdo con los mejores intereses de ella. Y ante la ausencia de otros familiares o personas cercanas, consultar la decisión al Comité de Bioética, justificando un paternalismo que apela al beneficio de la paciente.

CI3: Dado el contexto familiar y los antecedentes de maltrato y la falta de competencia de ella para tomar decisiones relativas a su internamiento, mantenerla en el INNN por un lapso de 30 días para evaluar su situación. Transcurrido ese plazo, tomar una decisión al respecto; además, notificar al Ministerio Público (MP).

CI4: En razón de la incompetencia de la paciente, atender la solicitud de su madre y aceptar que vaya a casa. En este caso, haciendo del co- nocimiento del MP los antecedentes de maltrato y abuso sexual de los que fue víctima, para que tome las medidas que considere oportunas, informándole tanto a ella como a su familia de esa notificación. Además, involucrar al área de trabajo social del INNN.

CI5: Atender la solicitud de su madre y aceptar que se vaya a casa sin informar a la autoridad del maltrato y abuso sexual, pero haciéndole hincapié que puede volver al INNN en cualquier momento, en caso de que vuelva a ser objeto de maltrato o abuso sexual.

CI6: No dar el alta voluntaria a Ángeles y remitirla a un albergue de víctimas de violencia, en donde cambie por algún tiempo su ambiente social y se le enseñen herramientas para construir otro camino de vida.

\section{Justificación del curso óptimo de acción}

Usualmente, el Curso Óptimo de Acción (COA) no se limita a uno solo de los cursos intermedios (CIA); incluso, se recomienda que sea una mezcla de varios de ellos, o bien que sean acciones que se emprendan sucesivamente. La riqueza moral y profesional de las personas que participan en la deliberación se refleja directamente en el número y calidad de cursos de acción propuestos. Para ello, es necesaria una sólida formación bioética, esto es, en la correcta gestión de valores morales en contextos y situaciones de incertidumbre, y una vasta experiencia profesional en distintas áreas del conocimiento que permitan descifrar y conocer los hechos relevantes del caso.

En este orden de ideas, considerando los múltiples problemas (no solo morales) del caso en particular, la importancia de los valores en conflicto y los CIA propuestos, el COA sería: informar al MP acerca de la violencia de la que ha sido víctima Ángeles, de la petición de alta del INNN y de la incompetencia para la toma de decisiones. Involucrar al área de trabajo social y al Comité de Bioética del INNN, así como al CAVI. Mientras se normaliza de algún modo su situación familiar $y$, sobre todo, el estado emocional, mantenerla internada, dando un lapso de 30 días para volver a valorar la situación ante el Comité de Bioética. 
Es muy importante informar al MP de la situación familiar de Ángeles. Aunque ella carece de competencia, legalmente aún tiene capacidad para tomar sus propias decisiones. En un sentido muy riguroso, mantenerla internada en contra de su voluntad podría constituir un delito, pero, si se justifica su internamiento conforme al principio de beneficencia, en virtud de su incompetencia, es aconsejable ponerlo en conocimiento de esa autoridad, para prever un conflicto legal para los médicos y/o autoridades del INNN.

Por otro lado, el INNN no puede arrogarse funciones de protección a víctimas de violencia intrafamiliar, aunque tampoco es moralmente aceptable aumentar su vulnerabilidad desentendiéndose de la paciente. Deben trabajar conjuntamente él o los médicos tratantes, trabajo social, el CAVI y el Comité de Bioética.

\section{Discusión}

Una reflexión se refiere a la necesidad de deliberación ética en situaciones clínicas infrecuentes, en las cuales aparecen conflictos morales. La sistematización de la medicina, favorecida por los esquemas del tecno-racionalismo capitalista o burocrático, tienden a llevar a una automatización de las decisiones clínicas, es decir, a una formación de protocolos y algoritmos rígidos y anónimos para la toma de decisiones, basados en la medicina por evidencia o en el consenso de los expertos. Sin embargo, aunque los protocolos de atención automatizados pueden ser útiles en la mayoría de las situaciones, diariamente acontecen problemas clínicos inéditos o infrecuentes que obligan a procesos de deliberación prudente, es decir, a lo que Gadamer llama "razonabilidad del saber práctico", para lo cual emplea el término griego phronesis, discutido por Aristóteles en la Ética, y traducido frecuentemente como "deliberación prudente"(15). En este sentido, la primera decisión relevante es presentar el caso en el Comité de Bioética, para no conceptualizarlo como un caso clínico más de "depresión", en el que se debe proteger al paciente de sus impulsos autodestructivos a toda costa, mientras se espera su curación mediante psicofármacos.
Uno de los problemas de este caso radica en la falta de eficacia de varios esquemas psicofarmacológicos antidepresivos que fueron empleados, así como la dificultad para clasificar a la paciente en cuestión como portadora de un "trastorno depresivo", de acuerdo con los estereotipos de la Asociación Psiquiátrica Americana(16), cuya imagen no es congruente con la fenomenología clínica de este caso. Más que un reduccionismo diagnóstico, el abordaje de Ángeles requiere una valoración integral de los problemas del entorno familiar y social, y de la historia de vida. En este sentido, la decisión ética adoptada (separar a la paciente de su ambiente familiar) se justifica desde el punto de vista psicoterapéutico, ya que el juicio clínico especializado en este caso informa que el principal factor generador de malestar profundo y "pérdida del sentido de vida" es la relación de maltrato y abuso por parte del padrastro. La deliberación clínica o ética pondera los aspectos particulares del caso que hacen difícil la aplicación de principios ideales de manera automática, ya que estos entran en conflicto en el caso particular. La postergación en el ejercicio de la libertad en el presente caso solo se justifica porque la valoración clínica informa acerca de una probable pérdida previa del sentido de libertad, debida a la coerción externa que llevó a Ángeles a un estado de indefensión aprendida y a una relación de dependencia emocional ambivalente con su victimario. En este contexto puede justificarse la actitud paternalista del equipo médico, pues representa una forma de paternalismo dialógico que se aplica en sustitución del paternalismo autoritario, incestuoso, encubierto y violento que fungía como la orientación esencial de su historia de vida.

La labor del INNN es de atención médica pública a significativos sectores de la población, orientada por los principios de beneficencia y justicia, los cuales pueden entrar en conflicto como en el caso presentado. Si bien la labor de hacer el bien y cuidar de los pacientes, junto con la no maleficencia, es el "santo y seña de medicina", según Diego Gracia, de igual manera existen límites que impone la realidad en cuanto a la capacidad de hacer el bien, señalados por la justicia. Por una parte, el INNN no cuenta con la infraestructura para cubrir el complejo conjunto de necesidades de cada paciente, no solo por sus recursos esca- 
sos, sino también por su identidad, que es ante todo la de una institución de asistencia médica y no solo de beneficencia. Además, por razones de justicia distributiva, el INNN está también obligado a dar lugar y atención a otros pacientes y es imposible detenerse de más en la atención de uno solo. Por supuesto que cada caso requiere de una valoración personalizada, pero atendiendo a principios éticos orientativos.

Es evidente que el proceso salud, enfermedad y atención trasciende el ámbito técnico médico. El caso en particular invita a examinar temas tan complejos como el derecho a la muerte, la sociogénesis de algunas enfermedades mentales, la concepción del Estado, la participación ciudadana, las facultades y obligaciones de los servicios médicos, entre otros.

Casos tan complejos dejan ver la insuficiencia del papel del Estado, que debería ser el principal agente de la justicia en la modernidad. Las privaciones de bienestar social, tales como la pobreza, la falta de educación y de atención de la salud, así como no reparar las violaciones a los derechos humanos, implican faltas a la justicia social.

Así, este espacio de reflexión no solo hace patente la importancia de la deliberación bioética en el ejercicio médico, también da cuenta de las limitaciones de orden moral y social que prevalecen en el contexto mexicano, caracterizado por la falta de interés en garantizar una vida digna y libre de violencia. El caso da cuenta de una vida de marginación absoluta: mujer, sin educación, sin recursos económicos, sin redes sociales, víctima de violencia doméstica. Es un itinerario sospechosamente común, que revela un sistema social permisivo con la violencia contra las mujeres, sustentado en relaciones de género inequitativas. Sumado a este contexto, es preocupante que no existan instituciones de atención a la violencia, que cuenten con infraestructura y recursos humanos capacitados para atender a personas que, además de sufrir violencia, padecen una enfermedad mental. El Estado está obligado no solo a prestar servicios para atender el maltrato sino que éstos sean eficientes $y$ eficaces. 
¿En qué circunstancias el paternalismo médico puede estar justificado? - Mariana Espínola-Nadurille et al.

\section{Referencias}

1. Sánchez-Barroso JA. Vigencia y operatividad de los principios en la bioética en la solución de problemas a partir de la deliberación moral y de la argumentación jurídica. Persona Bioética 2010; 14(2): 187-204.

2. Beauchamp TL, Childress JF. Principios de ética biomédica. Barcelona: Masson; 2001.

3. Sánchez-Barroso JA. Influencia de los principios de la bioética en la elaboración y aplicación del derecho. Buenos Aires: RAP; 2011.

4. Simón P. El consentimiento informado. Madrid: Tricastela; 2000.

5. Drane JF. Clinical bioethics. Kansas City: Sheed \& Ward; 1994.

6. Lazar NM, Greiner GG, Robertson G, Singer P. Bioethics for clinicians: substitute decision-making. Can Med Assoc 1996; 155(10): 1435-1437.

7. Atienza M. Discutamos sobre paternalismo. Doxa 1988; 5: 203-214.

8. Durkheim E. El suicidio. Madrid: Akal; 1995.

9. Cohen-Agrest D. Por mano propia: estudio sobre las prácticas suicidas. Buenos Aires: Fondo de Cultura Económica; 2010.

10. Reza H. Suicidio lúcido y patológico. Psiquis 2009; 18(1): 23-28.

11. O’Neill O. Agents of Justice. En: Pogge T, (edit.) Global Justice. EEUU: Blackwell Publising; 2000: 88-203.

12. Sen A. The Idea of Justice. Harvard, Cambridge, Massachusetts: The Belknap Press; 2009.

13. Miller BL. Autonomy. En: Encyclopedia of Bioethics. NY, EUA: McMillan; 2004: 246-2451.

14. Battin MP. Suicide. En: Encyclopedia of Bioethics. NY, EUA: Mc Millan; 2004: 2475-2483.

15. Gadamer HG. El último dios: la lección del siglo XX. Un diálogo filosófico con Ricardo Dottori. México: Anthropos; 2010.

16. Asociación Psiquiátrica Americana. DSM-IV-TR. Manual diagnóstico y estadístico de los trastornos mentales. Madrid: Masson; 2009.

Recibido: 16 de octubre de 2012

Aceptado: 18 de diciembre de 2012 